\title{
Salud, calidad de vida y consumo de sustancias en función del tiempo en situación sin hogar
}

\author{
Sonia Panadero-Herrero* y Manuel Muñoz-López
}

Universidad Complutense de Madrid

\begin{abstract}
Resumen: Este artículo tiene como objetivo principal conocer las características diferenciales de aquellas personas que están "recién" llegadas a la situación sin hogar frente a aquellas que llevan un largo tiempo en dicha situación. Concretamente en este artículo se presentan los resultados de la comparación en diferentes variables (calidad de vida, salud, etc.) entre personas sin hogar que llevaban menos de un año en esa situación y personas sin hogar que llevaban más de 5 años, con la intención de profundizar en el efecto de la situación sin hogar e identificar variables diferenciales que permitan avanzar en la identificación de necesidades específicas, favoreciendo así la planificación de servicios y recursos ajustados a las necesidades de cada grupo y momento. Los resultados apuntan diferencias importantes entre ambos grupos que parecen indican un mayor deterioro entre aquellos que llevan más tiempo en la situación sin hogar (mayor consumo de alcohol y otras sustancias, más discapacidades, etc.) así como unas mayores carencias (duermen en mayor medida literalmente en la calle, ejercen en mayor medida la mendicidad, etc.).

Palabras clave: Personas sin hogar; cronicidad; calidad de vida; salud; consumo de sustancias.
\end{abstract}

\section{Introducción}

Aunque en general se aborda el estudio de las personas sin hogar (PSH) como un conjunto homogéneo, cada vez con mayor frecuencia se reconoce la existencia de diferentes subgrupos dentro de este colectivo (Levinston, 2004; Muñoz, Panadero, Pérez y Quiroga, 2005). De hecho, el fallo a la hora de reconocer grupos diferenciados dentro del colectivo sin hogar ha dado lugar en muchas ocasiones a un inadecuado programa de servicios sociales y de salud. Es decir, la identificación y caracterización de subgrupos dentro del colectivo sin hogar resulta muy útil a la hora de responder de una manera efectiva al fenómeno sin hogar, concretamente a la hora de diseñar las políticas e intervenciones.

Una vez que se reconoce la posible existencia de subgrupos entre las PSH, toma un especial interés la investigación de las características y necesidades de los mismos. La postura más clásica adoptada para la identificación de estos subgrupos es la identificación de los mismos a partir de algunas características de las personas como el sexo o la edad. Algunos de los subgrupos cuyas características y necesidades diferenciales han despertado un mayor interés han sido las mujeres sin hogar (Chitvil, 2010; Marsapt, 2000), las personas sin hogar con trastornos mentales (Lee et al., 2010; Sullivan, Burnam, y Koegel, 2000; Cohen y Thompson, 1992) y los veteranos sin hogar (e.g. Mares y Rosenheck, 2006; Rosenheck y Koegel, 1993), aunque se han utilizado otras muchas variables para identificar o caracterizar subgrupos dentro de las personas sin hogar: edad (Salavera, Puyuelo, y Orejudo, 2009; Washington, Moxley y Taylor, 2009; DeMai-

* Dirección para correspondencia [Correspondence address]: Sonia Panadero. Facultad de Psicología. UCM. Campus de Somosaguas, s/n. 28223. Madrid (España). E-mail: spanadero@psi.ucm.es
Title: Health, Quality of life and substances consume and length of homelessness.

Abstract: The study investigated main characteristics of "new" homeless people vs. "old" or "chronic" homeless people. Specifically this paper presents the differences in relevant variables (quality of life, health, alcohol and drug consume) between homeless people who were homeless less than one year and those who were more than five years in this situation. Aim of that is to investigate the effect of length of homelessness and identify differential variables that permit to recognize specific needs and planning services and resources for each group and moment. Results show important differences between both of groups: higher deterioration among long-term homeless (higher alcohol and drugs consume, disabilities) and more difficulties (more rough sleepers, begging, etc.).

Key words: Homeless; quality of life; health; substance abuse.

lle, North y Smith, 1997), tiempo sin hogar (e.g. Rickards et al., 2010; Arce y Vergara, 1994) o lugar en el que duermen (e.g. Larsen, Poortinga y Hurdle, 2004; Roth y Bean, 1986).

A lo largo de los últimos años también han aparecido trabajos que se han interesado por las características diferenciales de las personas sin hogar que se "cronifican" en dicha situación, así como sobre las implicaciones que tiene sobre esas personas pasar un largo periodo de tiempo en esta situación. Así, se han identificado algunos factores de riesgo para la cronicidad de la situación sin hogar como el abuso durante la infancia (Stein, Leslie and Nyamaty, 2002) o un mayor número de arrestos (Caton, Domínguez, Schanzer, y Hasin, 2005). Por otro lado, se ha relacionado la cronicidad sin hogar con el consumo de drogas (Baron, 1999; Rodríguez, 1995; Tyler y Johnson, 2006), una mayor mortalidad asociado a un mayor número de enfermedades y discapacidades (Barrow, 1999) y trastornos mentales (North, Pollio, Smith y Spitznagel, 1998; Wenzel, Gelberg, Barkhtiar, Caskey et al., 1993; Rodríguez, 1995). Por otro lado, un estudio longitudinal de Caton et al., (2005) señaló que la menor duración en la situación sin hogar estaba asociada a una menor edad, empleo actual o reciente, recibir algún tipo de ingresos económicos, buenas habilidades de afrontamiento, adecuado apoyo familiar, ausencia de historia de abuso de sustancias y ausencia de historia de arrestos.

Los estudios españoles sobre población sin hogar han tendido a considerar a las personas sin hogar como un grupo homogéneo, aunque el interés por identificar las características y necesidades particulares de los subgrupos dentro del colectivo sin hogar ha ido aumentando a lo largo del tiempo.

Reconociendo la importancia de la investigación de las características y necesidades de los subgrupos dentro del colectivo sin hogar, en España se han realizado algunos traba- 
jos que han explorado algunos de estos subgrupos como inmigrantes (Cabrera, 2002; Muñoz, Vázquez y Vázquez, 2003), mujeres (Cabrera, 2000) o jóvenes (Romero, Ramos y March, 2002).

Sin embargo, hay muy pocos trabajos que se hayan interesado por las peculiaridades de las personas sin hogar en función del tiempo en dicha situación. Este artículo tiene como objetivo principal conocer las características diferenciales de aquellas personas que están "recién" llegadas a la situación sin hogar frente a aquellas que llevan un largo tiempo en dicha situación. Concretamente en este artículo se presentan los resultados de la comparación en diferentes variables (calidad de vida, salud, autoestima, etc.) entre personas sin hogar que llevaban menos de un año en esa situación y personas sin hogar que llevaban más de 5 años, con la intención de profundizar en el efecto de la situación sin hogar e identificar variables diferenciales que permitieran avanzar en la identificación de necesidades específicas, favoreciendo así la planificación de servicios y recursos ajustados a las necesidades de cada grupo y momento.

\section{Método}

\section{Participantes}

Los participantes en este estudio, realizado en la ciudad de Madrid, fueron 130 usuarios de 11 programas previamente seleccionados dirigidos a la atención de personas sin hogar. Estos programas fueron seleccionados por un grupo de expertos por ajustarse a los criterios de buenas prácticas utilizados en el proyecto "To Live in Health and Dignity" (H\&D) (Craig, Brandt, Leonori, y Muñoz, 2002) que aparecen recogidos en la Tabla 1.

Tabla 1. Criterios de buenas prácticas (a partir del Proyecto H\&D).

1. El usuario es el foco central del programa.

2. El programa debe tener una aproximación integral global.

3. Los métodos de aproximación y acercamiento dignos, no humillantes, son prioritarios.

4. Atención a la persona completa, con necesidades complejas.

5. Asistencia a la medida de las necesidades específicas de los clientes (atención individualizada).

6. Capacidad para responder de manera flexible a las diferentes necesidades.

7. Tener la posibilidad de intervención de urgencia y en situaciones de crisis.

8. Colaboración entre programas de otras instituciones.

9. Equipos multidisciplinares.

10. Participación de voluntarios no profesionales.

11. Asociación e interdisciplinariedad, tanto dentro como fuera del programa.

12. Integración y colaboración en la red local

13. Supervisión y entrenamiento continuo de los profesionales.

14. Compromiso de la sociedad civil.

Fueron considerados no aptas para ser incluidas en el estudio aquellas personas con un dominio del castellano insuficiente para realizar la entrevista o que no estaban en condiciones de aportar una información fiable durante la misma, es decir, presentaban un discurso incoherente, ya fuera por trastorno mental o por encontrarse bajo los efectos del alcohol u otras sustancia psicoactivas.

De los 130 participantes, teniendo en cuenta los objetivos del artículo, tan sólo se consideraron en este caso los datos de aquellos que llevaban menos de un año sin hogar (45 personas) y aquellos que llevaban en dicha situación más de 5 años (41 personas); es decir, en este artículo se presentan los datos de un total de 86 personas sin hogar. Las características sociodemográficas de estos participantes eran muy similares a las encontradas en otros estudios españoles previos: mayoritariamente varones (el $88.5 \%$ de los participantes), con una edad media de $44.54(D T=11.45)$, de nacionalidad española $(87.7 \%$ ) y solteros $(58.5 \%)$.

\section{Procedimiento}

El contacto con los participantes se llevó a cabo a través de los profesionales de los diferentes programas. Todas las entrevistas se realizaron de forma anónima protegiendo la privacidad de los datos.

El instrumento utilizado en este estudio fue aplicado en forma de entrevista estructurada, para facilitar la comprensión de los usuarios e incluyó diversos dominios e instrumentos. En este artículo se incluyen datos sobre los siguientes aspectos:

- Calidad de vida. Para la evaluación de este dominio se utilizó el Quality of Life Interview (Lehman, 1993), entrevista estructurada que mide la calidad de vida objetiva y subjetiva (en su versión abreviada de 78 items). Evalúa 8 áreas básicas: residencia, relaciones familiares, relaciones sociales, ocio, actividades cotidianas y funcionamiento, finanzas, seguridad y problemas legales, trabajo y salud.

- Salud. Se decidió utilizar el General Health Questionnaire (GHQ-28; Goldberg, 1996) que evalúa el estado de salud general de la persona. Se centra en el deterioro del funcionamiento normal, más que en los rasgos presentes a lo largo de toda la vida. En esta ocasión se utilizó la versión de 28 items.

- Consumo de alcohol y otras sustancias. En este caso la mayoría de las preguntas han sido realizadas de forma específica para este estudio y en otros casos han sido tomadas de 
otros estudios previos, y se centran en recoger información sobre la frecuencia y cantidad de consumo de diferentes sustancias.

\section{Análisis de datos}

Para realizar el proceso de análisis de datos se utilizó el sistema de análisis estadístico y de gestión de datos SPSS (versión 15 para Windows).

Para establecer las diferencias entre aquellos participantes que llevaban menos de un año sin hogar y aquellos que llevaban más de 5 años en esa situación se utilizó: el estadístico Chi-cuadrado para variables nominales y pruebas " $t$ " de Student para muestras independientes en el caso de las variables continuas.

\section{Resultados}

De las 130 personas que fueron entrevistadas en el curso del estudio, 45 llevaban en la situación sin hogar menos de un año y otras 41 personas se encontraban en dicha situación desde hacía más de cinco años. A continuación se presentan los resultados de la comparación realizada entre ambos grupos.

En primer lugar se presenta la comparación entre las variables sociodemográficas de ambos grupos, cuyos resultados aparecen recogidos en la Tabla 2. Como puede verse, no aparecieron diferencias estadísticamente significativas en cuanto a la proporción de hombres y mujeres (alrededor de $10 \%$ de mujeres $)(\chi 2(1)=.250, p=.617)$ y el estado civil (más de la mitad, solteros) $(\chi 2(4)=1.889, p=.756)$. Especialmente llamativa resulta la no existencia de diferencias significativas entre los dos grupos en edad (en torno a los 44 años, en ambos casos) $(t(84)=0.372, p=0.711)$. Sin embargo, sí aparecen diferencias estadísticamente significativas en el origen de los participantes $(\chi 2(1)=9.814, p=.002)$ : aunque en ambos grupos la mayoría de los participantes eran españoles, en el grupo de aquellos que llevaban menos de un año sin hogar uno de cada cuatro aproximadamente eran extranjeros, mientras que entre aquellos que llevaban más de 5 años el porcentaje se redujo, hasta el 2,4\%. También aparecen diferencias significativas respecto al nivel educativo de los participantes, mayor en aquellos que llevan poco tiempo $\sin \operatorname{hogar}\left(\chi^{2}(1)=15.969, p=.025\right)$.

Tabla 2. Características sociodemográficas de los participantes.

\begin{tabular}{|c|c|c|c|c|c|}
\hline & \multicolumn{2}{|c|}{$0-12$ meses } & \multicolumn{2}{|c|}{$>60$ meses } & \multirow{2}{*}{$\frac{\chi^{2} / t(1)}{0.250}$} \\
\hline Sexo & & & & & \\
\hline Hombres & 41 & $91.1 \%$ & 36 & $87.8 \%$ & \\
\hline Mujeres & 4 & $8.9 \%$ & 5 & $12.2 \%$ & \\
\hline Edad (Media (DT)) & 45 & $43.42(11.297)$ & 41 & $44.29(10.313)$ & -0.372 \\
\hline Nacionalidad & & & & & $9.814^{* *}$ \\
\hline Española & 33 & $73.3 \%$ & 40 & $97.6 \%$ & \\
\hline No española & 12 & $26.7 \%$ & 1 & $2.4 \%$ & \\
\hline Estado civil & & & & & 1.889 \\
\hline Soltero & 26 & $57.8 \%$ & 24 & $58.5 \%$ & \\
\hline Casado & 4 & $8.9 \%$ & 3 & $7.3 \%$ & \\
\hline Divorciado o separado legalmente & 10 & $22.2 \%$ & 12 & $29.3 \%$ & \\
\hline Separado de hecho & 4 & $8.9 \%$ & 2 & $4.9 \%$ & \\
\hline Viudo & 1 & $2.2 \%$ & 0 & 0 & \\
\hline Estudios & & & & & $15.969 *$ \\
\hline Sin estudios, no lee & 2 & $4.4 \%$ & 1 & $2.4 \%$ & \\
\hline Sin estudios, lee & 2 & $4.4 \%$ & 5 & $12.2 \%$ & \\
\hline Primarios incompletos & 5 & $11.1 \%$ & 0 & 0 & \\
\hline Primer grado (EGB $1^{\text {a }}$ etapa, ingreso, hasta los 10 años) & 5 & $11.1 \%$ & 6 & $14.6 \%$ & \\
\hline Segundo grado, primer ciclo (EGB, $2^{\mathrm{a}}$ etapa, $4^{\circ}$ bachiller, secretariado, etc.) & 13 & $28.9 \%$ & 19 & $46.3 \%$ & \\
\hline Segundo grado, segundo ciclo (BUP, Bachiller superior, FP2, etc.) & 6 & $13.3 \%$ & 8 & $19.5 \%$ & \\
\hline Tercer grado (diplomados, técnicos, licenciados, doctores) & 12 & $26.6 \%$ & 2 & $4.9 \%$ & \\
\hline
\end{tabular}

\section{(1) Se han aplicado pruebas $t$ para muestras independientes para las variables continuas y pruebas de $\chi^{2}$ para las variables categóricas} ${ }^{*} p \leq .05 ;{ }^{* *} p \leq .01{ }^{* * *} p \leq .001$

Respecto al lugar en el que pernoctaron los participantes en el estudio en el mes previo a la realización de la entrevista, aspecto considerado en el Quality of Life Interview (Lehman, 1993), como se observa en la Tabla 3, sólo aparecieron diferencias significativas en el porcentaje de personas de cada grupo que durmieron en la calle en el mes anterior a la realización de la entrevista $(\chi 2(1)=3.873, p=.049)$. El 36.6\% de aquellos que llevaban más de cinco años sin hogar habían dormido al aire libre, directamente en la calle, mientras que el caso de aquellos que llevaban menos de un año este porcentaje se redujo al $17.8 \%$. Sin embargo, no aparecieron diferencias estadísticamente significativas en el porcentaje de personas que durmieron en un edificio vacío o similar, ni en un albergue. 
Tabla 3. Situación de alojamiento en el último mes (Quality of Life Interview; Lehman, 1993).

\begin{tabular}{lccccc}
\hline & \multicolumn{2}{c}{$0-12$ meses } & \multicolumn{2}{c}{$>$} & $\chi^{2}$ \\
\cline { 2 - 6 } & $n$ & & $n$ & \\
Durmió al aire libre & 8 & $17.8 \%$ & 15 & $36.6 \%$ & $3.873 *$ \\
Durmió en un edificio vacío o similar & 6 & $13.3 \%$ & 7 & $17.1 \%$ & 0.234 \\
Durmió en un albergue público & 26 & $57.8 \%$ & 18 & $43.9 \%$ & 1.653 \\
Tiene actualmente un sitio regular para vivir & 36 & $80.0 \%$ & 32 & $78.0 \%$ & 0.049 \\
\hline
\end{tabular}

${ }^{*} p \leq .05 ;{ }^{* *} p \leq .01 ; * * * \leq .001$

La frecuencia del contacto con la familia de los participantes de ambos grupos, recogida mediante el Quality of Life Interview, se presenta en la tabla siguiente. Como puede observarse no aparecieron diferencias significativas ni en con- tacto telefónico ni personal con los miembros de su familia entre aquellos que llevaban más de cinco años sin hogar y aquellos que llevaban menos de un año en esa situación.

$\underline{\text { Tabla 4. Frecuencia del contacto con la familia. }}$

\begin{tabular}{|c|c|c|c|c|c|}
\hline & \multicolumn{2}{|c|}{$0-12$ meses } & \multicolumn{2}{|c|}{$>60$ meses } & \multirow[t]{2}{*}{$\chi^{2}$} \\
\hline & $n$ & & $n$ & & \\
\hline $\begin{array}{l}\text { En el último mes ¿con qué frecuencia habló por teléfono con un } \\
\text { miembro de su familia? }\end{array}$ & & & & & 4.726 \\
\hline Sin familia & 3 & $6.7 \%$ & 2 & $5.0 \%$ & \\
\hline Nunca & 14 & $31.1 \%$ & 21 & $52.5 \%$ & \\
\hline Menos de una vez al mes & 7 & $15.6 \%$ & 5 & $12.5 \%$ & \\
\hline Al menos una vez al mes & 9 & $20.0 \%$ & 6 & $15.0 \%$ & \\
\hline Al menos una vez por semana & 11 & $24.4 \%$ & 6 & $15.0 \%$ & \\
\hline Al menos una vez al día & 1 & $2.2 \%$ & 0 & $0.0 \%$ & \\
\hline $\begin{array}{l}\text { En el último mes, ¿con qué frecuencia estuvo con un miembro } \\
\text { de su familia? }\end{array}$ & & & & & 1.518 \\
\hline Sin familia & 3 & $6.7 \%$ & 1 & $2.5 \%$ & \\
\hline Nunca & 25 & $55.6 \%$ & 21 & $52.5 \%$ & \\
\hline Menos de una vez al mes & 9 & $20.0 \%$ & 8 & $20.0 \%$ & \\
\hline Al menos una vez al mes & 4 & $8.9 \%$ & 4 & $10.0 \%$ & \\
\hline Al menos una vez por semana & 2 & $4.4 \%$ & 3 & $7.5 \%$ & \\
\hline Al menos una vez al día & 2 & $4.4 \%$ & 3 & $7.5 \%$ & \\
\hline
\end{tabular}

${ }^{*} p \leq .05 ;{ }^{* *} p \leq .01 ; * * * p \leq .001$

Mediante la Quality of Life Interview, también se recogió información sobre el tipo de ingresos económicos y la cuantía de los mismos en el mes previo a la realización de la entrevista. Como se recoge en la Tabla 5, los ingresos de las personas sin hogar que participaron en este estudio procedían principalmente de un trabajo (en torno a un 15\%), de la Renta Mínima de Inserción (casi una de cada tres personas que llevaban más de cinco años sin hogar) y del ejercicio de la mendicidad. Tan sólo aparecieron diferencias estadísticamente significativas respecto al ejercicio de la mendicidad, ejercida en mayor medida por aquellos que llevaban más de cinco años sin hogar $(\chi 2(1)=12.230, p \leq .000)$. El $29.3 \%$ de las personas de ese grupo habían ejercido la mendicidad. Sobre la cantidad de los ingresos económicos percibidos, no aparecieron diferencias estadísticamente significativas, situándose los ingresos medios de ambos grupos por debajo de los 300 euros $(t(81)=-1.942, p=.056)$.

Respecto a la situación laboral de los entrevistados no aparecieron diferencias estadísticamente significativas $(\chi 2(1)$ $=0.004, p=.953)$ : en ambos grupos casi el $70 \%$ no habían trabajado durante el último mes ni se encontraba trabajando en el momento de la entrevista, lo que indica las importantes carencias de esta población en este sentido.

Para analizar la salud de los participantes en el estudio se aplicó el GHQ (Goldberg, 1996) cuyas puntuaciones en las diferentes escalas quedan recogidas en la Tabla 6. Las puntuaciones más altas en los dos grupos aparecieron en la Escala de ansiedad e insomnio, en el que el grupo que llevaba menos de un año sin hogar presentaba una peor puntuación. Mientras estas personas presentaban una puntuación media de $2.73(D T=2.13)$, el grupo de participantes que llevaban más de cinco años en esta situación presentaban una puntuación media de $1.55(D T=1.88)$. Esa misma tendencia aparece en la escala de disfunción social, en la que parecen encontrarse peor aquellos que llevan menos tiempo en la situación sin hogar. En las escalas de síntomas somáticos y depresión, las puntuaciones entre ambos grupos son muy similares, así como en la puntuación total del GHQ. 
Tabla 5. Tipo de ingresos económicos, cantidad recibida y suficiencia para cubrir diferentes necesidades.

\begin{tabular}{|c|c|c|c|c|c|}
\hline & \multicolumn{2}{|c|}{$0-12$ meses } & \multicolumn{2}{|c|}{$>60$ meses } & \multirow[t]{2}{*}{$\chi^{2 / t}(1)$} \\
\hline & $n$ & & $n$ & & \\
\hline \multicolumn{6}{|l|}{ En el pasado mes ha recibido... } \\
\hline un sueldo & 8 & $17.8 \%$ & 6 & $14.6 \%$ & 0.156 \\
\hline alguna prestación social no contributiva & 1 & $2.2 \%$ & 5 & $12.2 \%$ & 3.288 \\
\hline pensión de invalidez & 1 & $2.2 \%$ & 1 & $2.4 \%$ & 0.004 \\
\hline empleo protegido & 2 & $4.4 \%$ & 4 & $9.8 \%$ & 0.933 \\
\hline paro & 3 & $6.7 \%$ & 1 & $2.4 \%$ & 0.865 \\
\hline jubilación, ahorros & 2 & $4.4 \%$ & 1 & $2.4 \%$ & 0.256 \\
\hline dinero de familiares $y / o$ esposo/a & 2 & $4.4 \%$ & 4 & $9.8 \%$ & 0.933 \\
\hline Renta Mínima de Inserción (RMI) & 7 & $15.6 \%$ & 13 & $31.7 \%$ & 3.136 \\
\hline mendicidad & 1 & $2.2 \%$ & 12 & $29.3 \%$ & $12.230^{* * *}$ \\
\hline Ingresos medios mensuales en euros $(D T)$ & 45 & $\begin{array}{l}212.24 \\
(205.98)\end{array}$ & 38 & $\begin{array}{l}296.71 \\
(186.76)\end{array}$ & -1.942 \\
\hline ¿Ha trabajado durante el último mes? ¿Está trabajando ahora? & & & & & 0.004 \\
\hline No trabajó el mes pasado ni está trabajando actualmente & 31 & $68.9 \%$ & 28 & $68.3 \%$ & \\
\hline Está trabajando o ha trabajado en el último mes & 14 & $31.1 \%$ & 13 & $31.7 \%$ & \\
\hline Tiempo medio sin empleo en meses $(D T)$ & 37 & $\begin{array}{l}54.89 \\
(140.33)\end{array}$ & 29 & $\begin{array}{l}116.31 \\
(176.13)\end{array}$ & -1.577 \\
\hline
\end{tabular}

(1) Se han aplicado pruebas t para muestras independientes para las variables continuas y pruebas de $\chi^{2}$ para las variables categóricas ${ }^{*} p \leq .05 ;{ }^{* *} p \leq .01 ; * * p \leq .001$

También se recogieron otros datos relacionados con la salud de los participantes, como la existencia de discapacidades y hospitalizaciones. El porcentaje de personas con discapacidad era significativamente mayor entre aquellos que llevaban más de 5 años sin hogar $\left(\chi^{2}(1)=5.207, p=.026\right)$; aproximadamente una de cada tres personas de ese grupo presentaban alguna discapacidad. Aproximadamente un 15\% habían estado hospitalizados en el mes previo a la realización de la entrevista, no encontrándose diferencias significativas entre los grupos.

Tabla 6. Puntuaciones medias en las escalas, puntuación total y positivos en el GHQ.

\begin{tabular}{|c|c|c|c|c|c|}
\hline & \multicolumn{2}{|c|}{$0-12$ meses } & \multicolumn{2}{|c|}{$>60$ meses } & \multirow[t]{2}{*}{$t$} \\
\hline & $n$ & & $n$ & & \\
\hline \multicolumn{6}{|l|}{ GHO } \\
\hline Escala A. Síntomas somáticos (media $(D T))$ & 44 & $1.93(1.97)$ & 40 & $1.72(1.95)$ & 0.483 \\
\hline Escala B. Ansiedad e insomnio (media $(D T)$ ) & 45 & $2.73(2.13)$ & 40 & $1.55(1.88)$ & $2.704^{* *}$ \\
\hline Escala C. Disfunción social (media $(D T))$ & 44 & $1.23(1.54)$ & 35 & $0.63(0.91)$ & $2.151 *$ \\
\hline Escala D. Depresión (media $(D T)$ ) & 45 & $0.91(1.49)$ & 37 & $0.92(1.85)$ & -0.021 \\
\hline $\begin{array}{l}\text { Puntuación total GHQ (media }(D T) \text { ) } \\
\text { Discapacidades }\end{array}$ & 43 & $6.63(4.95)$ & 34 & $5.15(5.37)$ & 1.256 \\
\hline $\begin{array}{l}\text { Padece alguna discapacidad física o sensorial } \\
\text { Hospitalizaciones }\end{array}$ & 6 & $13.3 \%$ & 14 & $34.1 \%$ & $5.207^{*}$ \\
\hline Ha estado hospitalizado en el último mes & 7 & $15.9 \%$ & 6 & $14.6 \%$ & 0.027 \\
\hline
\end{tabular}

${ }^{*} p \leq .05 ;{ }^{* *} p \leq .01 ; * * * p \leq .001$

La Tabla 7 recoge información sobre el consumo de alcohol de las personas de ambos grupos. En este caso sí aparecieron diferencias significativas en el consumo de alcohol de los dos grupos $\left(\left(\chi^{2}(4)=19.190, p \leq .001\right)\right.$, más frecuente entre aquellos que llevaban más tiempo en la situación: aproximadamente la mitad de las personas de este grupo habían bebido casi a diario durante el último mes. Sin embargo, menos del $10 \%$ de las personas que llevaban menos de un año habían consumido con esta frecuencia. Por otro lado, mientras la mitad de las personas que llevaban menos de un año habían bebido alcohol menos de una vez al mes (durante los últimos 30 días), este porcentaje se reducía al 20\% entre aquellos que llevaban más de cinco años.
Como resulta esperable en vista de lo anterior el consumo medio diario de ambos grupos también fue significativamente diferente $[(t(63.185)=-2.217, p=.030) ; 7.49(D T$ $=12.05)$ vasos de media en el caso de aquellos que llevaban más de cinco años y $2.71(D T=7.038)]$ entre los que llevaban menos de un año sin hogar. Respecto al consumo de otras sustancias, aparecieron diferencias significativas en el consumo de heroína, cannabis y sedantes en el último mes. Las personas que llevaban más tiempo sin hogar tenían un consumo más importante de estas sustancias (un 19.5\% habían consumido heroína, el $31.7 \%$ cannabis y un $34.1 \%$ sedantes) significativamente mayor que aquellos que llevaban menos de un año. 
Tabla 7. Consumo de alcohol y otras sustancias.

\begin{tabular}{|c|c|c|c|c|c|}
\hline & \multicolumn{2}{|c|}{$0-12$ meses } & \multicolumn{2}{|c|}{$>60$ meses } & \multirow{2}{*}{$\frac{\chi^{2} / t(1)}{19.190^{* * *}}$} \\
\hline En el último mes, ha bebido aunque, fuera poco,: & & & & & \\
\hline casi a diario & 4 & $8.9 \%$ & 20 & $48.8 \%$ & \\
\hline 3 ó 4 días a la semana & 3 & $6.7 \%$ & 3 & $7.3 \%$ & \\
\hline 1 ó 2 días a la semana & 7 & $15.6 \%$ & 6 & $14.6 \%$ & \\
\hline de 1 a 3 días al mes & 8 & $17.8 \%$ & 4 & $9.8 \%$ & \\
\hline menos de 1 vez al mes & 23 & $51.1 \%$ & 8 & $19.5 \%$ & \\
\hline Media vasos alcohol diarios $(D T)$ & 45 & $\begin{array}{l}2.71 \\
(7.038)\end{array}$ & 41 & $\begin{array}{l}7.49 \\
(12.05)\end{array}$ & $-2.217^{*}$ \\
\hline \multicolumn{6}{|l|}{ En los últimos 30 días ha consumido... } \\
\hline Cocaína & 5 & $11.1 \%$ & 11 & $26.8 \%$ & 3.500 \\
\hline Heroína & 2 & $2.4 \%$ & 8 & $19.5 \%$ & 4.740* \\
\hline Cannabis & 5 & $11.1 \%$ & 13 & $31.7 \%$ & $5.499 *$ \\
\hline Sedantes & 7 & $15.6 \%$ & 14 & $34.1 \%$ & $4.740^{*}$ \\
\hline
\end{tabular}

(1) Se han aplicado pruebas t para muestras independientes para las variables continuas y pruebas de $\chi 2$ para las variables categóricas $* p \leq .05 ; * * p \leq .01 ; * * p \leq .001$

\section{Discusión y conclusiones}

Este artículo presenta los resultados de la comparación de las características diferenciales de dos grupos de personas sin hogar en función del tiempo en esta situación. Parece razonable suponer la existencia de diferencias entre aquellas personas que acaban de quedarse sin hogar o llevan poco tiempo en esta situación y aquellos que llevan largo tiempo en dicha situación. Este artículo ha pretendido indagar en dichas características diferenciales comparando personas sin hogar que llevaban menos de un año en dicha situación y aquellos que llevaban más de 5 años. La comparación de ambos grupos ha mostrado algunas diferencias pero también muchas similitudes.

En primer lugar, ambos grupos resultan similares en algunas de sus características sociodemográficas como el sexo y el estado civil de los participantes. Es interesante señalar que, a pesar de las diferentes definiciones y metodologías utilizadas en los diferentes trabajos, las características sociodemográficas de los dos grupos de participantes en estos estudios fueron muy similares a las encontradas en otros estudios españoles previos (e.g. Vega, 1996; Cabrera, 1998; Muñoz et al, 2003; INE, 2005), así como en estudios internacionales (Helvie y Kunstmann, 1999; Marsapt, 1999; Toro, 1998), lo que confirma la constancia de algunas características entre las personas sin hogar: predominantemente varones, con una edad media entre 37 y 45 años y solteros.

Sin embargo, también se observaron algunas diferencias entre las personas que llevaban sin hogar menos de un año y aquellas personas sin hogar de larga duración: el porcentaje de personas sin hogar de origen extranjero entre estos últimos era mucho menor que entre aquellos "recién" llegados a la situación sin hogar. Entre los participantes que llevaban más de 5 años sin hogar el porcentaje de inmigrantes era casi nulo, mientras que suponía casi uno de cada cuatro de aquellos "recién" llegados a la situación sin hogar. La mayor presencia de personas inmigrantes en los colectivos más recientes y su práctica desaparición de los servicios a los 5 años y su disminución de presencia en la calle puede ser explicada por dos factores concomitantes. En primer lugar, el estudio recoge datos de un momento en el que si se contabilizan más de 5 años antes la presencia de inmigrantes en nuestro país era menor; aún así la disminución es tan notable que obliga a buscar otras explicaciones.

En segundo lugar, la explicación puede venir dada por el hecho de que los inmigrantes que están sin hogar lo están principalmente por motivos económicos y sus estrategias sociales, recursos personales y redes de apoyo están más conservados, lo que facilita una salida relativamente rápida de la situación sin hogar, especialmente cuando son ayudados por los servicios sociales, de ahí su escasa presencia en esta situación a largo plazo. En esta misma línea, se encontrarían los trabajos de algunos autores que han identificado algunos subgrupos dentro del colectivo sin hogar y que parecen apuntar en el mismo sentido (Muñoz, Panadero, Pérez y Quiroga, 2005).

Además de las características sociodemográficas se recogió también información sobre diferentes aspectos de la vida: situación de alojamiento, laboral, económica, relación familiar, salud y consumo de alcohol y otras sustancias. Los resultados en general dibujan una muy difícil situación de los participantes. Por ejemplo, los datos apuntan a una precaria situación laboral, similar en ambos grupos: casi un 70\% no habían trabajado en el último mes y llevaban de media entre 5 (en el grupo que llevaba menos de un año sin hogar) y 10 años (en el grupo que llevaba más de 5 años sin hogar). En el caso de la salud aparecieron algunos datos preocupantes, lo que coincide con lo encontrado en otros estudios (Baron, 1999; Instituto de Salud Pública de Madrid, 2006). Resulta especialmente preocupante el porcentaje de discapacidades registrado entre el grupo que llevaban más tiempo en la situación sin hogar (a pesar de presentar una edad similar al del otro grupo): uno de cada tres participantes que llevaban más de 5 años en esta situación tenía algún tipo de discapacidad. Por otro lado, también aparecen algunos resultados que muestran una peor situación de salud en el grupo que llevaba menos tiempo sin hogar. Concretamente, el grupo que llevaba un menor tiempo en la situación sin hogar mos- 
traba peores puntuaciones en dos de las escalas del GHQ, las de Ansiedad e insomnio y Disfunción social. Este cuestionario evalúa cambios en diferentes aspectos relacionados con la salud durante el último mes. Por lo tanto, estos resultados pueden estar indicando que en el grupo que llevaba menos de 12 meses sin hogar se estaba produciendo un empeoramiento de su situación de salud probablemente por la necesidad de adaptarse a la nueva situación; por ejemplo, es esperable que existan problemas de sueño teniendo en cuenta los lugares en los que estas personas dormían (albergues, calle,...) cuyas características pueden dificultar el sueño, al menos al principio. Por otro lado, en el caso de las personas que llevaban más de 5 años en la situación la situación estaba más estable o el deterioro era más lento probablemente por su adaptación a las condiciones de vida que implica la situación sin hogar.

Especialmente precaria parece la situación de estas personas en aquellos aspectos relacionados con el alojamiento, la situación económica y la relación familiar, en los que se apunta un deterioro muy importante. Por ejemplo, respecto a los ingresos económicos medios de ambos grupos eran inferiores a los 300 euros y en el caso de las personas sin hogar "crónicas" usuarias de recursos provenían en una parte importante del ejercicio de la mendicidad, lo que no ocurría en el caso de los que llevaban menos de un año sin hogar. En este grupo el ejercicio de la mendicidad era algo muy minoritario.

A pesar de que en ambos grupos las relaciones familiares estaban deterioradas en el caso de las personas que llevaban más de 5 años sin hogar la satisfacción con esas relaciones era significativamente menor, a pesar de no encontrarse diferencias entre ambos grupos respecto a la frecuencia del contacto.

Respecto a la situación de alojamiento, los datos confirman los serios problemas en este aspecto de los participantes: alrededor de la mitad habían dormido en un albergue público y alrededor de un $15 \%$ en un edificio vacío o similar. La situación de alojamiento, parece peor en el caso de aquellos que llevaban más tiempo sin hogar: más de uno de cada tres habían dormido al aire libre en el último mes.

En la línea de lo encontrado en trabajos previos (Baron, 1999; Rodríguez, 1995; Tyler y Johnson, 2006), el aspecto en el que se aprecian más diferencias entre ambos grupos sea en los aspectos relacionados con el consumo de alcohol y otras sustancias. El consumo de alcohol fue mucho más habitual entre el grupo que llevaba más de 5 años en la situación sin hogar, según aparece en ambos estudios.

En resumen, los datos parecen apuntar hacia una caracterización del colectivo de personas sin hogar recientes como con alta presencia de inmigrantes y un importante papel de los problemas económicos como principal estresor. Los grupos de más larga duración se caracterizan por la presencia de graves problemas de salud física y mental, de consumo de alcohol y otras drogas, de discapacidad y con pobres relaciones sociales y muy escasos recursos personales. En estos grupos se observa una menor presencia de inmigrantes (no justificada exclusivamente por los momentos temporales de los muestreos) que cuando se cronifican parecen tener unas características muy similares a las de los españoles en las variables estudiadas.

A la vista de los datos resumidos hasta el momento puede concluirse que existen diferencias en función del tiempo en la situación sin hogar que justifican un acercamiento diferencial en función del tiempo de permanencia en la calle de las personas. Estas diferencias deberían ser tenidas en cuenta a la hora de diseñar programas de intervención que respondan de forma específica a las necesidades diversas y cambiantes de las personas sin hogar en función de sus problemas y necesidades y del tiempo de permanencia en dicha situación. Así, parece fundamental que la intervención se organice en función de dos ejes principales: tipos de problemas principales y tiempo en la calle.

El trabajo expuesto se suma a otros anteriores que destacan la importancia del alcohol en el proceso rehabilitador y como factor predictor de la cronicidad. El trabajo expuesto se suma a otros anteriores que destacan la importancia del alcohol en el proceso rehabilitador y como factor predictor de la cronicidad. Esto apunta a la necesidad de potenciar las acciones dirigidas al control del consumo de alcohol, desde su prevención hasta la intervención en el colectivo más crónico, acciones necesariamente fundamentadas en los planes individualizados de intervención. Esto hace necesario que la intervención con estas personas sea llevada a cabo en recursos de alojamiento de larga duración con un número muy reducido de plazas (aproximadamente 20-30 plazas), con profesionales de diferente tipo (social, pero también sanitario) especialmente profesionales formados en tratamiento de alcohol, drogas y discapacidad y un abordaje de estos problemas adecuado a este colectivo y a la gravedad del problema (por ejemplo, programas de reducción del daño). Resultarían muy útiles, para facilitar el acceso de aquellas personas que se encuentran literalmente en la calle durante años, los recursos de baja exigencia que contemplen la dificultad de adaptarse a unas normas rígidas de estas personas que llevan largo tiempo sin hogar. No habría que olvidar el trabajo para fomentar la autonomía de estas personas, así como los hábitos más básicos para la vida independiente: asumir responsabilidades en la elaboración de su comida, lavado de ropa, higiene personal, etc.

Para finalizar, debe advertirse que los resultados de este trabajo provienen de un diseño transversal que dificulta en gran medida la generalización de resultados y la interpretación del verdadero papel del tiempo en el proceso sin hogar. Resultaría importante a la hora de elaborar estrategias de prevención e intervención eficaces llevar a cabo investigaciones de tipo longitudinal, muy escasas hasta el momento en nuestro entorno, que nos ayudasen a identificar factores de riesgo para la cronicidad, entre las personas que llegan por primera vez a la situación sin hogar y las alternativas de intervención social, psicológica, de salud mental y de salud más efectivas. 


\section{Referencias}

Arce, A. y Vergare, M. (1984). Identifying and characterizing the mentally ill among the homeless. En H.R. Lamb (Ed.), Identifying and characterizing the mentally ill among the homeless. Washington, D.C: American Psychiatric Association.

Baron, S. (1999). Street youths and substance use. The role of background, street lifestyle and economic factors. Youth and Society, 3-26. doi: 10.1177/0044118X99031001001.

Cabrera, P. (1998). Huéspedes del aire: Sociología de las personas sin hogar en Madrid. Madrid: UPCO.

Cabrera, P. (2000). Mujeres sin hogar en España. Informe Nacional para FEANTSA

Cabrera, P. (2002). Inmigrantes y sinhogarismo en España. Informe Nacional 2001-2002 para FEANTSA.

Caton, C., Domínguez, B., Schanzer, B., Hasin, D. S. (2005). Risk factors for long-term homelessness: findings from a longitudinal study of first-time homeless single adults. American journal of public bealth, 95, 10. doi: 10.2105/AJPH.2005.063321

Chityil, B. (2010) Homelessness in Europe: The Role of Gender Equility Policies. Homeless in Europe, spring, 4-5.

Cohen, C.I. y Thompson, K.S. (1992). Homeless mentally ill or mentally ill homeless? American Journal of Psychiatry, 149, 816-823.

Craig, T., Brandt, P., Leonori, L. y Muñoz, M. (2002). To live in bealth and dignity. European report of study and action project in promotion of mental health and social reinsertion for disadvantaged people. Copenhague: Udenfor.

DeMallie, D. A., North, C. S. y Smith, E. M. (1997). Psychiatric disorders among the homeless: A comparison of older and younger groups. The Gerontologist, 37(1), 61-66. doi: 10.1093/geront/37.1.61.

Goldberg, D. P. (1996). Cuestionario de salud general de Goldberg. Barcelona: Masson.

Helvie, C. O. y Kunstmann, W. (1999). Comparison of definitions, prevalence, demographics, trends, causes and health concerns in seven countries. En C. O. Helvie y W. Kunstmann (Eds.), Homeless: An international perspective. Westport, C.T: Greenwood Press.

INE (2005). Encuesta sobre las personas sin hogar.

Instituto de Salud Pública de Madrid (2006). Investigación sobre la percepción que los "grupos vulnerables" de la ciudad de Madrid tienen de su saludy sus condicionantes. Madrid.

Larsen, L., Poortinge, E. y Hurdle, D. E. (2004). Sleeping rough: Exploring the differences between shelter-using and non-shelter-using homeless individuals. Environment and Behavior, 36, 578-591. doi: $10.1177 / 0013916503261385$.

Lee, S., DeCastella, A., Freidin, J., Kroschel, J., Humphrey, C., kroschel, J., Humphrey, C., Kerr, R., Hollows, A., Wilkins, S., Kulkarni, J., et al. (2010). Mental health care on the streets: An integrated approach. Australian and New Zealand Journal of Psychiatry, 44, 505-512.

Lehman, A. F. (1988). A Quality research of Life Interview for the chronically mentally ill. Evaluation and program planning, 11, 51-62.

Mares, A. S. y Rosenheck, R. A. (2006). Attitudes towards employment and employment outcomes among homeless veterans with substance abuse and/or psychiatric problems. American Journal of Psychiatric Rehabilitation, 9, 145-166. doi: 10.1080/15487760600961451.

Marsapt, M. (1999). Les sans-domicile à Paris et aux Etats Unis. Données Sociales, 480-489.
Marsapt, M. (2000). An advantage with limits. The lower risk for women of becoming homeless. Population: An English selection, 12, 1-45.

Muñoz, M., Panadero, S., Pérez Santos, E. y Quiroga, M. A. (2005). Role of stressful life events in homelessness: An Intragroup análisis. American Journal of Community Psychology, 35 (1/2), 35-47. doi: 10.1007/s10464-0051888-6.

Muñoz, M., Vázquez, C. y Vázquez, J. J. (2003). Los límites de la exclusión: Estudio sobre los factores económicos, psicosociales y de salud que afectan a las personas sin hogar en Madrid. Madrid: Editorial Témpora.

North, C., Pollio, D. E., Smith, E. M. y Spitznagel, E. L. (1998). Correlates of early onset and chronicity of homelessness in a large urban homeless population. Journal of nervous and mental disease, 186(7), 393-400. doi: 10.1097/00005053-199807000-00002.

Rickards, L. D., McGraw, S. A., Araki, L., Casey, R. J., High, C. W., Hombs, M. E. y Rayson, R. S. (2010). Collaborative initiative to help end chronic homelessness: Introduction. The Journal of Behavioral Health Services \& Research, 37, 149-166. doi: 10.1007/s11414-009-9175-1.

Rodríguez, G. (1995). Treatment modality for the chronic homeless: A case study. Doctoral dissertation.

Romero, M., Ramos, M. y March, J. C. (2002). Perfil de los jóvenes sin hogar, dificultades para la utilización de los servicios sociosanitarios y propuestas de mejora. Enf Emerg, 4(1), 14-23.

Rosenheck, R. y Koegel, P. (1993). Characteristics of veterans and nonveterans in three simples of homeless men. Hospital and Community Psychiatry, 44(9), 858-862.

Roth, D. y Bean, J. (1986). The Ohio study: A comprehensive look at homelessness. Psychosocial rehabilitation journal, 9(4), 31-38.

Salavera, C., Puyuelo, M. y Orejudo, S. (2009). Trastornos de personalidad y edad: Estudio con personas sin hogar. Anales de psicología, 25(1), 261265.

Stein, J. A., Leslie, M. B. and Nyamathi, A. (2002). Relative contributions of parent substance use and childhood maltreatment to chronic homelessness, depression, and substance abuse problems among homeless women: mediating roles of self-esteem and abuse in adulthood. Child Abuse and Neglect, 26, 1011-1027. doi: 10.1016/S0145-2134(02)00382-4.

Sullivan, G., Burnam, A. y Koegel, P. (2000). Pathways to homelessness among the mentally ill. Social psychiatry and psychiatric epidemiology, 35, 444450.

Toro, P. A. (1998). Homeless. En S. Bellock y M. Hersen (Eds.), Comprehensive Clinical Psychology. Elservier Science.

Tyler, K y Johnson, K. (2006). Pathways in and out of substance use among homeless-emerging adults. Journal of adolescent research, 21(2), 133-157. doi: $10.1177 / 0743558405285494$.

Vega, L. S. (1996). Salud mental en población sin hogar: Estudio epidemiológico en albergues para transeúntes de Gijón (Asturias). Oviedo: Sespa.

Washington, O. G. M., Moxley, D. P. y Taylor, J. Y. (2009). Enabling older homeless minority women to overcome homelessness by using a life management enhancement group intervention. Issues in Mental Health Nursing, 30, 86-97. doi: 10.1080/01612840802597580.

Wensel, S., Gelberg, L., Bakhtiar, L., Caskey, N, et al. (1993). Indicators of chronic homelessness among veterans. Hospital and community psychiatry, 44(12), 1172-1176.

(Articulo recibido: 13-10-2011; revisado: 25-01-2013; aceptado: 06-02-2013) 